\title{
Delivery of Complex Organic Compounds from Planetary Nebulae to the Solar System
}

\author{
Sun Kwok \\ Faculty of Science, The University of Hong Kong, Pokfulam Road, Hong Kong \\ e-mail:sunkwok@hku.hk
}

\begin{abstract}
Infrared spectroscopic observations of planetary nebulae and proto-planetary nebulae have shown that complex organic compounds are synthesized in these objects over periods as short as a thousand years. These compounds are ejected into the interstellar medium and spread throughout the Galaxy. Evidence from meteorites has shown that these stellar grains have reached the Solar System, and may have showered the Earth during the heavy bombardment stage of the Early Earth. In this paper, we discuss the chemical structure of stellar organic grains and compare them to the organic matter found in meteorites, comets, asteroids, planetary satellites, and interplanetary particles. The possibility that the early Solar System was chemically enriched by organic compounds ejected from distant stars is presented.

Received 22 March 2009, accepted 17 April 2009, first published online 26 May 2009
\end{abstract}

Key words: chemical synthesis, infrared spectroscopy, meteorites, molecular spectroscopy, origin of life, stellar evolution.

\section{Introduction}

Since the Miller-Urey experiment in 1953 (Miller 1953; Miller \& Urey 1959), the endogenous origin of organic matter has remained popular for 50 years. The Miller-Urey experiment was seen by the scientific community as the experimental confirmation of the ideas of Oparin (1938) and Haldane (1929) that origin of life on the early Earth can be explained using only the laws of chemistry and physics. Although the idea of panspermia has been around for some time (Arrhenius 1908), it has never found much support except by the works of Hoyle and Wickramsinghe (see, e.g., Hoyle \& Wickramsinghe 1977). This perception changed, however, with the discovery of extensive presence of organic compounds in primitive meteorites, specifically carbonaceous chondrites (Cronin \& Chang 1993), raising the possibility that some of the organic matter on Earth may have been delivered from extraterrestrial sources. From the crater records of the Moon, we learn that the Earth has suffered from heavy bombardment (Kring 2008) and a large amount of extraterrestrial carbon and other minerals was likely to have been delivered to the Earth from comets, asteroids, and interplanetary dust particles (Chyba et al. 1990; Chyba \& Sagan 1992).

The development of high-frequency radio receivers and their application in millimetre and submillimetre-wave astronomical spectroscopy have made possible the detection of over 140 gas-phase molecules in the interstellar medium, the majority of which are organic molecules (Ehrenfreund \&
Charnley 2000, for a complete current list, see http://aramis. obspm.fr/mol/list-mol.html). These detections clearly show that organic molecules of moderate complexity can be synthesized beyond the terrestrial environment, even under extreme low-density conditions. However, there is a limit to the degree of complexity for molecules to be detected through their rotational transitions. As the number of energy levels increases with the complexity of the molecule, the relative population in each of the rotational states decreases, therefore producing a weaker line. Even with increasing instrument sensitivity, there is a limit at which every part of the millimetre and submillimetre-wave spectrum is filled with weak lines of common abundant molecules (such as methanol), therefore crowding out lines of larger molecules and making the detection of transitions from less abundant complex molecules difficult.

Recent advances in infrared spectroscopy, in particular from space-based observatories, have opened up the possibility of detecting the stretching and bending modes of complex molecules. These observations reveal the presence of organic compounds consisting of hundreds of atoms, with definite aromatic and aliphatic structures (Pendleton \& Allamandola 2002). Most interestingly, we now have evidence that these compounds are formed over a very short time ( $\left.\sim 10^{4} \mathrm{yr}\right)$ during the late stages of stellar evolution. In this paper, we will summarize the evidence for the synthesis and chemical evolution of organic compounds, and discuss their possible relationship to similar compounds found in meteorites. 


\section{Evolved stars as molecular factories}

The discovery of large-scale mass loss in asymptotic giant branch (AGB) stars in the 1970s led to the realization that the majority of stars in the Milky Way Galaxy (stars with initial masses between 1 and $8 \mathrm{M}$, corresponding to $\sim 95 \%$ of stars with lifetimes within the age of the Galaxy) can shed most of their envelope mass and avoid the fate of ending their lives as supernovae (Kwok 1987). The mass loss process takes place in the form of a steady stellar wind over the last $\sim 10^{6}$ years of the AGB evolution, and manifests itself through line emission from molecules in the wind, as well as continuum infrared emission from solid-state particles condensed in the wind. Near the end of the AGB, the ejection rate can be so large that the solid particles in the ejecta completely shield the stellar photosphere from the view of the observer, and these stars can only be seen in infrared wavelengths. Depending on whether the photospheric chemical composition has more oxygen than carbon, the stars with $\mathrm{O} / \mathrm{C}>1$ are mostly discovered either in $\mathrm{OH}$ surveys (known as $\mathrm{OH} / \mathrm{IR}$ stars), and those with $\mathrm{O} / \mathrm{C}<1$ by mid-infrared surveys (known as extreme carbon stars).

As of early 2009, over 60 gas-phase molecules have been detected in the circumstellar envelopes of AGB stars. These include inorganics (e.g., $\mathrm{CO}, \mathrm{SiO}, \mathrm{SiS}, \mathrm{NH}_{3}, \mathrm{AlCl}$, etc), organics $\left(\mathrm{C}_{2} \mathrm{H}_{2}, \mathrm{CH}_{4}, \mathrm{H}_{2} \mathrm{CO}, \mathrm{CH}_{3} \mathrm{CN}\right.$, etc), radicals $\left(\mathrm{CN}, \mathrm{C}_{2} \mathrm{H}\right.$, $\mathrm{C}_{3}, \mathrm{HCO}^{+}$, etc), chains (e.g., $\mathrm{HCN}, \mathrm{HC}_{3} \mathrm{~N}, \mathrm{HC}_{5} \mathrm{~N}$, etc), and rings $\left(\mathrm{C}_{3} \mathrm{H}_{2}\right)$. These molecules are detected primarily through millimetre/submillimetre spectroscopic observations of their rotational transitions, with some species detected through infrared spectroscopic of their vibrational bands in absorption against the infrared continuum.

Solid-state particles (commonly referred to as dust in the astronomical literature) also form from direct condensation from the gas phase. The most commonly detected solid particle is amorphous silicates (in oxygen-rich stars) and $\mathrm{SiC}$ in carbon-rich stars (Kwok et al. 1997; Henning 2009). A variety of refractory oxides (corundum $\left(\alpha-\mathrm{Al}_{2} \mathrm{O}_{3}\right)$, spinel $\left(\mathrm{MgAl}_{2} \mathrm{O}_{4}\right)$, rutile $\left.\left(\mathrm{TiO}_{2}\right)\right)$ are also present (Posch et al. 1999, 2002). For highly evolved carbon-rich stars, the dust component is believed to be dominated by amorphous carbon, which emits a strong featureless continuum in the infrared (Volk et al. 2000).

\section{Synthesis of complex organics in post-AGB evolution}

The discovery of the unidentified infrared emission bands in the planetary nebula NGC 7027 (Russell et al. 1977) and their subsequent identification as emissions from the stretching and bending modes of various $\mathrm{CH}$ and $\mathrm{CC}$ bonds in aromatic compounds (Duley \& Williams 1981) has led to the realization that complex organics can be synthesized in the circumstellar environment. Since planetary nebulae are a short-lived ( $\sim 20000 \mathrm{yr})$ phenomenon and the aromatic infrared bands (AIB) are not seen in the spectra of their progenitor AGB stars, it suggests that these aromatic compounds must have been synthesized in a short time under very low-density conditions.

The key to the understanding of the origin of the aromatic compounds in planetary nebulae lies in the observations of the transition objects between AGB stars and planetary nebulae, commonly referred to as proto-planetary nebulae. Protoplanetary nebulae have been a missing link in the late stages of stellar evolution as they are very difficult to detect due to the lack of emission lines as the circumstellar material is yet to be photoionized. Their discovery was made possible by the Infrared Astronomical Satellite (IRAS) all-sky infrared survey. By comparing the infrared properties of AGB stars and planetary nebulae, it is possible to infer the infrared properties of proto-planetary nebulae and search for their optical counterparts among the IRAS sources that fit the criterion (Volk \& Kwok 1989; Kwok 1990). The discovery of $\sim 30$ proto-planetary nebulae (Kwok 1993, an example is shown in Fig. 1) and their subsequent observations by the Infrared Space Observatory (ISO) have led to conclusion that aromatic compounds develop shortly after the AGB (Hrivnak et al. 2000). Most significantly, ISO spectra of proto-planetary nebulae show evidence for aliphatic chains attached to the aromatic rings (Fig. 2). Signatures of the aliphatic component take the form of the $3.4 \mu \mathrm{m} \mathrm{CH}$ stretching modes and $6.9 \mu \mathrm{m}$ in-plane-bending mode of the methyl $\left(-\mathrm{CH}_{3}\right)$ and methylene $\left(-\mathrm{CH}_{2}\right)$ groups, and the 8 and $12 \mu \mathrm{m}$ broad emission plateaus from a mixture of aliphatic side groups (Kwok et al. 2001). The presence of the 12.1, 12.4, and $13.3 \mu \mathrm{m}$ out-of-plane bending modes also suggests that the aromatic units in proto-planetary nebulae are smaller in size (with a corresponding larger number of exposed hydrogen edges) than those in planetary nebulae (Kwok et al. 1999).

Since the transition time between the end of AGB to the planetary nebulae stage is only several thousand years (Schönberner 1983; Kwok 2000), the synthesis of aromatic and aliphatic compounds must occur on a time scale shorter than this evolutionary time. It is interesting to note that the spectral signature of acetylene $\left(\mathrm{C}_{2} \mathrm{H}_{2}\right)$ only appears near the end of the AGB (Fig. 3), and benzene is detected in only one proto-planetary nebula, AFGL 618 (Cernicharo et al. 2001). We may therefore reasonably assume that aromatics evolved from the linear hydrocarbon acetylene which folds into a ring as benzene, which in turn attach with other rings into aromatics.

How such a reaction can take place in the gas phase under extremely low-density $\left(\sim 10^{6} \mathrm{~cm}^{-3}\right)$ conditions is difficult to understand theoretically. The process may resemble that of soot production in flames where gas-phase molecules first nucleate into nanometre size particles and then coalesce and grow into grains of thousands of atoms (Harris \& Weiner 1985). This scenario is supported by the fact that polyacetylenes first appear in the post-AGB phase of evolution. In Fig. 4 we show the ISO spectrum of the proto-planetary nebula AFGL 618, where diacetylene $\left(\mathrm{C}_{4} \mathrm{H}_{2}\right)$ and triacetylene $\left(\mathrm{C}_{6} \mathrm{H}_{2}\right)$ are detected in addition to acetylene. These polyacetylenes probably contribute towards the size growth as 


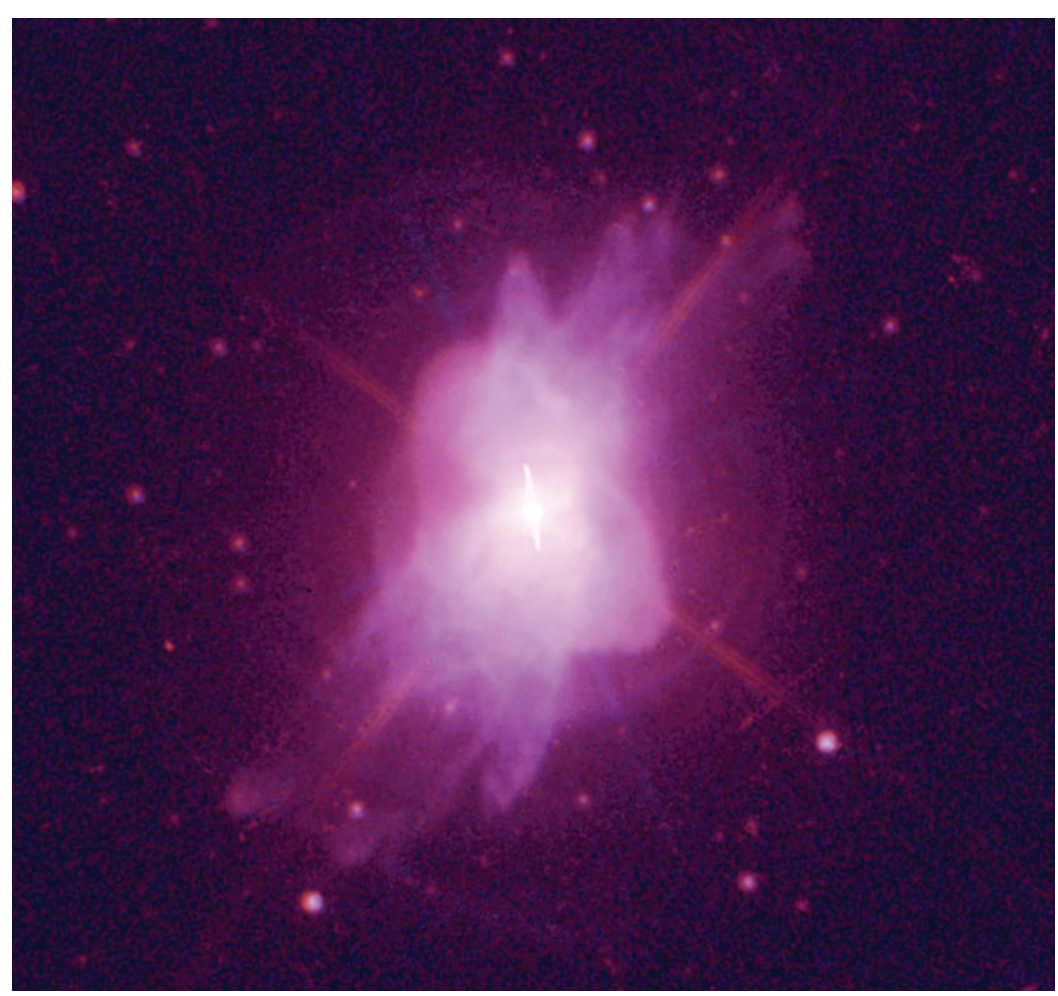

Fig. 1. Colour composite image of HST WFPC 2 I and V band observations of IRAS 16594-4656 (Water Lily Nebula, Hrivnak et al. 1999), a proto-planetary nebula that shows AIB emissions (Garcia-Lario et al. 1999).

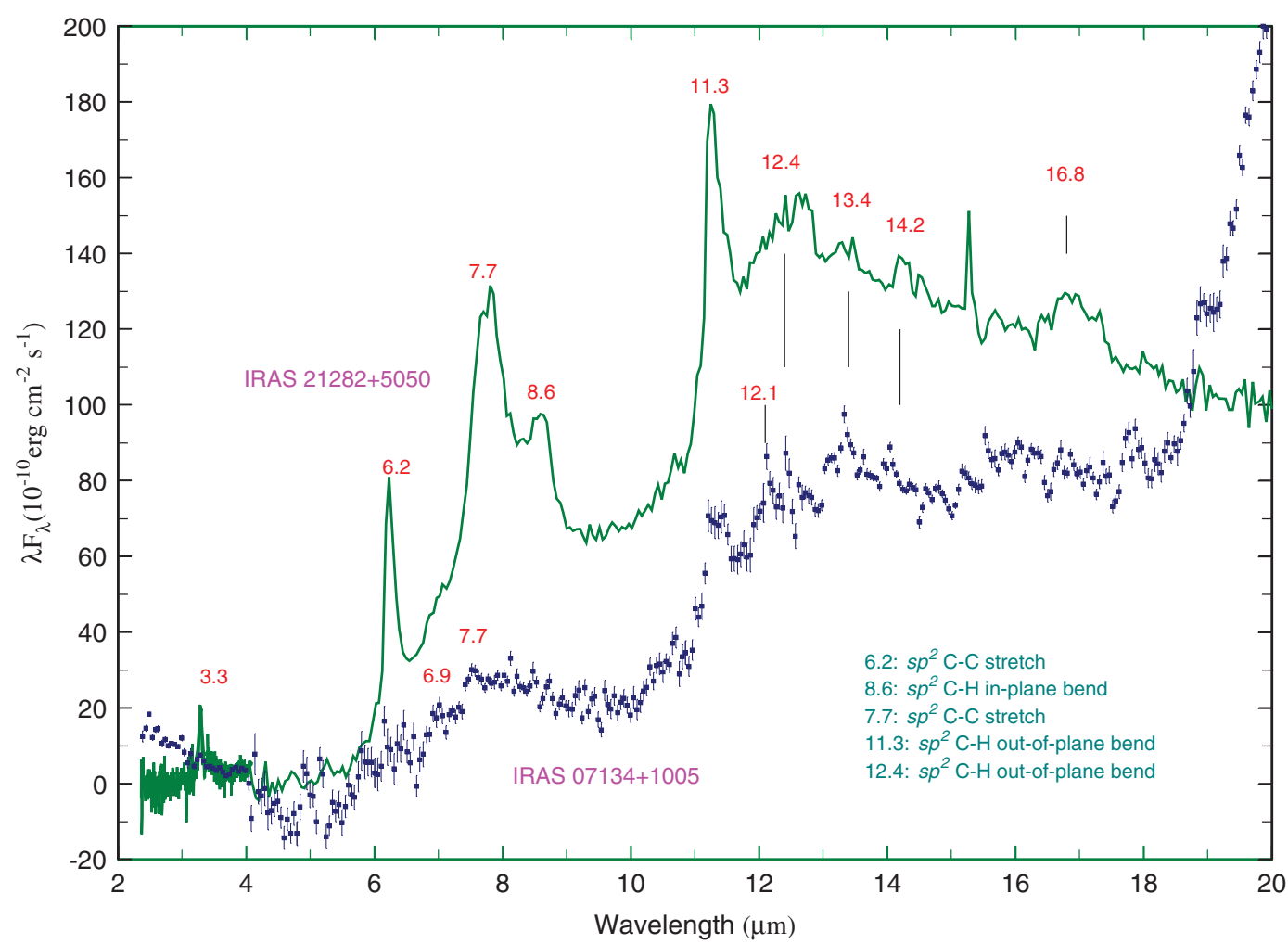

Fig. 2. ISO SWS 01 spectra of the young planetary nebula IRAS $21282+5050$ and the proto-planetary nebula IRAS $07134+1005$, showing various aromatic $\mathrm{C}-\mathrm{H}$ and $\mathrm{C}-\mathrm{C}$ stretching and bending modes at 3.3, 6.2, 7.7, 8.6, and $11.3 \mu \mathrm{m}$. The proto-planetary nebulae spectra are characterized by the $12.1,12.4,13.3 \mu \mathrm{m}$ out-of-plane bending mode features from small aromatic units. 


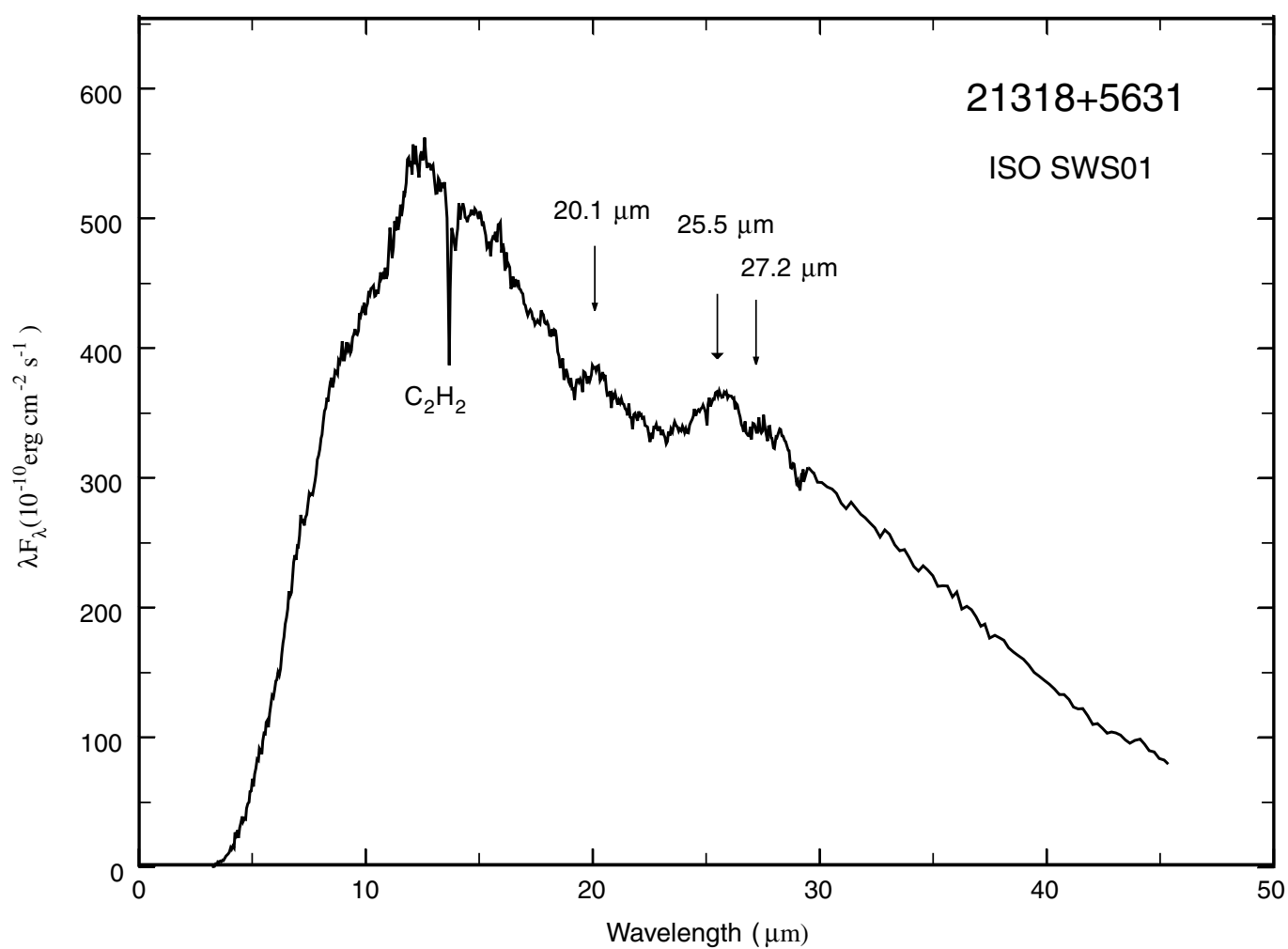

Fig. 3. The $13.7 \mu \mathrm{m} v_{5}$ vibrational band of acetylene is the strongest absorption feature in the ISO SW01 spectrum of the extreme carbon stars such as IRAS $21318+5631$. Extreme carbon stars are highly evolved AGB stars with such a high mass loss rate that circumstellar dust completely obscures the stellar photosphere and the starlight is re-emitted in the infrared through dust emission. Other features in the spectrum are the 21 and $30 \mu \mathrm{m}$ unidentified emission features.

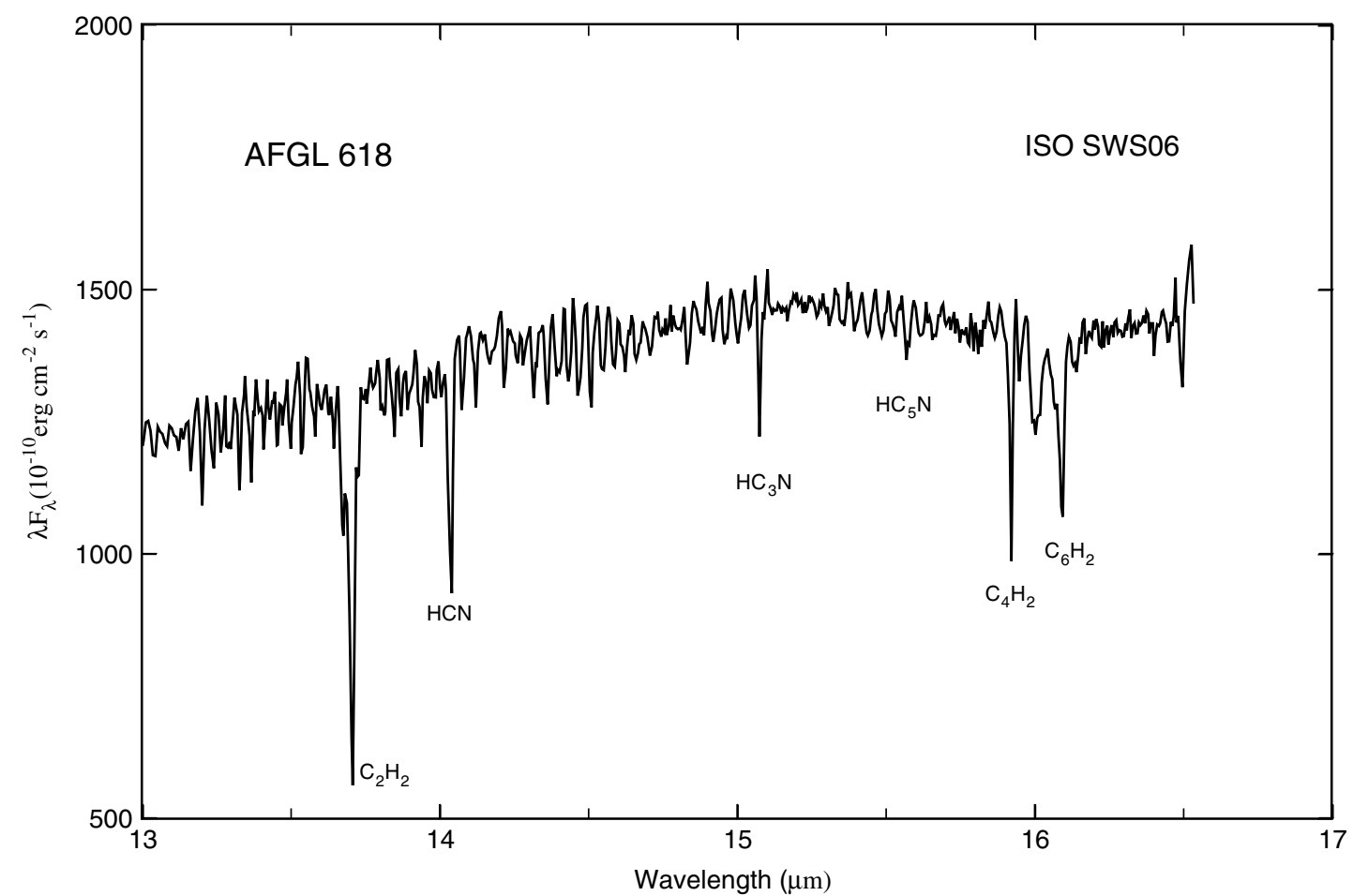

Fig. 4. ISO SWS06 spectrum of the proto-planetary nebula AFGL 618. In addition to the $13.7 \mu \mathrm{m}$ acetylene $\left(\mathrm{C}_{2} \mathrm{H}_{2}\right)$ feature, vibrational features of diacetylene $\left(\mathrm{C}_{4} \mathrm{H}_{2}\right)$ and triacetylene $\left(\mathrm{C}_{6} \mathrm{H}_{2}\right)$ can also be seen. 
well as the aromatization of the grains in the post-AGB phase of evolution.

\section{Chemical structure of the carrier of the aromatic infrared bands}

The observed spectral characteristics of proto-planetary nebulae suggest that the chemical structure of the carrier must consist of some aromatic rings, but probably in units of a small number of rings. These units are connected with each other by aliphatic bridges, which can be of diverse nature. The overall structure is probably disorganized and not highly structured. A possible schematic of the structure is given by Pendleton \& Allamandola (2002), although the actual structure may contain more aliphatics than they outlined. Spectral similarities between proto-planetary nebulae and terrestrial coal and kerogen have been pointed out by Guillois et al. (1996) and Papoular (2001). Kerogen differs from other amorphous hydrocarbons such as hydrogenated amorphous carbon (HAC, Jones et al. 1990) and quenched carbonaceous composites (QCC, Sakata et al. 1984, 1987) in that kerogen contains impurities such as $\mathrm{O}, \mathrm{N}$, and $\mathrm{S}$, whereas HAC and QCC are pure hydrocarbons. Under circumstellar conditions, it is natural to assume that any condensates will include other heavy elements as $\mathrm{N}$ can easily substitute $\mathrm{C}$ as one member of the aromatic structure. Such nitrogen heterocycles can be clearly identified when higher spectral resolution observations are available (Hudgins et al. 2005), and their detection would have significant biochemical implications.

Various techniques have been employed to artificially create carbon nanoparticles. These include condensation of carbon vapour from arc discharge between two carbon rods in an $\mathrm{H}_{2}$ atmosphere (Mennella et al. 1996), laser pyrolysis of gas-phase hydrocarbons (Herlin et al. 1998), and laser ablation of graphite and subsequent condensation under high temperatures (Jäger et al. 2008). The 1-4 nm size fullerenelike particles created by Jäger et al. (2008) show strong a $3.4 \mu \mathrm{m}$ aliphatic $-\mathrm{C}-\mathrm{H}$ stretching band feature as well as a $6.2 \mu \mathrm{m}$ aromatic $-\mathrm{C}=\mathrm{C}-$ stretching feature, suggesting the existence of aliphatic bridges between aromatic subunits. These onion structure grains could represent the first solid condensate in proto-planetary nebulae.

In summary, the carbonaceous compound made in the circumstellar envelopes of proto-planetary nebulae is likely to be a solid-state material made up of islands of aromatic rings, connected by aliphatic chains. In addition to hydrogen, functional groups (including those containing heavy elements $\mathrm{O}$ and $\mathrm{N})$, such as methyl $\left(-\mathrm{CH}_{3}\right)$, methylene $\left(-\mathrm{CH}_{2}\right)$, carbonyl $(\mathrm{C}=\mathrm{O})$, aldehydic $(-\mathrm{HCO})$, phenolic $(\mathrm{OH})$, and amine $\left(\mathrm{NH}_{2}\right)$, can be attached to the peripherals of these aromatic units. These structures are subjected to radiation processing as the star evolves to the planetary nebulae stage with an accompanying increasing amount of ultraviolet radiation output. This could result in consolidation of the rings and aromatization of the structure. To what extent these chemical structures are further processed by interstellar radiation field once they are ejected into the interstellar medium is uncertain.

\section{Relations between circumstellar and Solar System organic compounds}

Solar System objects display many spectral features suggesting that they contain similar minerals and organic matter as circumstellar grains. Both amorphous and crystalline silicates have been detected in the infrared spectra of comets (Wooden et al. 1999). It has been known for over 20 years that carbonaceous meteorites contain an insoluble macromolecular solid component often referred to as insoluble organic matter (IOM, Cronin et al. 1987). IOM is predominantly aromatic in structure but contains various functional groups (Kerridge 1999; Cody \& Alexander 2005) not dissimilar to the chemical structure of the organic solids found in proto-planetary nebulae. From the colour of asteroids, it is inferred that they may be covered by layers of polymer-type organic compounds which are structurally similar to kerogen (Gradie \& Veverka 1980; Cruikshank 2008).

X-ray absorption near-edge structure spectroscopy and infrared spectroscopy of interplanetary dust particles have revealed the presence of aliphatic $\mathrm{CH}_{2}$ and $\mathrm{CH}_{3}$, carbonyl $(\mathrm{C}=\mathrm{O})$ groups (Flynn et al. 2003). The $3.4 \mu \mathrm{m} \mathrm{CH}$ stretching bands observed in the spectra of meteorites (Ehrenfreund et al. 1991) is similar to those seen in proto-planetary nebulae (Hrivnak et al. 2007). These features are also seen in the spectra of the Saturnian satellites, Iapetus, Phoebe, and Hyperion, by the Visible-Infrared Mapping Spectrometer on board the Cassini spacecraft (Cruikshank et al. 2008).

\section{From planetary nebulae to the Solar System}

While it is now widely recognized that planetary nebulae and proto-planetary nebulae are major sources of organic matter in the Milky Way Galaxy, it has been argued that these stellar grains will be subjected to destruction by interstellar shocks and radiation field and therefore unable to survive the journey through the diffuse interstellar medium (Jones et al. 1996). The discovery of pre-solar grains of diamonds (Lewis et al. 1987), SiC (Bernatowicz et al. 1987), corundum (Nittler et al. 1997), spinel, etc in meteorites and the tracing of the origin of these grains to AGB stars through isotopic analysis has led to the amazed realization that one can actually hold a piece of star dust in our hands. Silicate grains of circumstellar origin have also been found in interplanetary dust particles (Messenger et al. 2003) and in meteorites (Nagashima et al. 2004; Nguyen \& Zinner 2004). The old paradigm of planets and other Solar System objects formed from a well-mixed primordial nebula of chemically and isotopically uniform composition and that no extrasolar solid could survive the formation of the Solar System (Suess $1965)$ is therefore no longer tenable.

Although there is no corresponding strong direct evidence of organic compounds having arrived from outside the Solar System, support for this hypothesis can be found in the fact that the organic compound $\mathrm{D} / \mathrm{H}$ ratios in some Solar System objects have non-solar values (Ehrenfreund et al. 2002; Ehrenfreund et al. 2006). The anomalous isotopic ratios of noble gases trapped in fullerenes $\left(\mathrm{C}_{60}\right.$ to $\left.\mathrm{C}_{400}\right)$ in the Allende 
and Murchison meteorites point to their extraterrestrial origin (Becker et al. 2000). The carbon isotope ratios of nucleobases in the Murchison meteorite also show signs that they are interstellar (Martins et al. 2008). The elevated ratios of ${ }^{15} \mathrm{~N} /{ }^{14} \mathrm{~N}$ and $\mathrm{D} / \mathrm{H}$ in organic globules in the Tagish Lake meteorite provide additional evidence that interstellar organics are present in the current Solar System (NakamuraMessenger et al. 2006).

\section{Effects of stardust on the origin of life}

The above observations have shown that planetary nebulae are capable of manufacturing complex organic compounds over very short time scales and ejecting them in large quantities into the interstellar medium. These organic compounds can migrate through the Milky Way Galaxy and have probably chemically enriched the early solar nebula. The subsequent mixing and formation of the Solar System have not completely destroyed the pre-solar materials and remnants of these organic solids are embedded in comets and other planetismals. Through the heavy bombardment episodes in the early days of the Solar System, these organics have likely been delivered to Earth from comets and asteroids. Macroscopic complex organics are much more likely than small molecules to have survived the impact.

If kerogen-like materials indeed represent the major constitutes of extraterrestrial organics, we may ask whether such organics played any role in the origin of life. Terrestrial kerogen is a solid sedimentary insoluble organic material found in the upper crust of the Earth. Since the fractions of $\mathrm{H}, \mathrm{S}, \mathrm{N}$, and $\mathrm{O}$ relative to $\mathrm{C}$ in kerogen are similar to those in lipids, they are believed to have formed as the result of decay of living organisms. Could it be the case that in the Early Earth, the process was reversed with fragments of extraterrestrial kerogen having served as suppliers of ingredients of the biomolecules that formed the basis of beginnings of life? While meteoritic IOM could lead to membrane structures (Deamer 1997), the emergence of genetic molecules such as DNA and RNA require nucleobases that are heterocyclic compounds heavy in $\mathrm{N}$ contents. Precursors of nucleobases such as purine and pyrimidine have large partition functions with their populations spread thinly over many rotational levels and therefore are difficult to detect in circumstellar environment by present millimetre-wave technology. The vibrational modes of $\mathrm{N}$-substituted aromatic units require high spectral resolution to be clearly identified. With the development of future large space-based spectroscopic telescopes, we are confident that such prebiotic molecules will be detected in time.

\section{Conclusions}

The discovery that evolved AGB stars and planetary nebulae are prolific molecular factories that are capable of rapid synthesis of complex organic molecules and solids has cast doubts on the completely endogenous theory of origin of life. The injection of extraterrestrial (and pre-solar) chemicals into the early Earth environment could play a role to facilitate or accelerate the development of life on Earth. Although the identification of circumstellar organics has so far been limited to hydrocarbons of aromatic and aliphatic structures, future improvements in sensitivity and spectral resolutions of space infrared telescopes can lead to the identification of $\mathrm{N}, \mathrm{P}$, and O substituted compounds that are essential elements of biochemistry.

\section{Acknowledgements}

I thank Bernard Foing and the organizers of the ESLAB Symposium on Cosmic Cataclysm and Life for a stimulating meeting. This work is supported by a grant from the Research Grants Council of the Hong Kong Special Administrative Region, China (Project No. HKU 7020/08P).

\section{References}

Arrhenius, S. (1908). Worlds in the Making. Harper, New York

Becker, L., Poreda, R.J. \& Bunch, T.E. (2000). Proc. Nat. Acad. Sci. 97, 2979-2983.

Bernatowicz, T., Fraundorf, G., Tang, M., Anders, E., Wopenka, B., Zinner, E. \& Fraundorf, P. (1987). Nature 330, 728-730.

Cernicharo, J., Heras, A.M., Tielens, A.G.G.M., Pardo, J.R., Herpin, F., Guélin, M. \& Waters, L.B.F.M. (2001). Astrophys. J. 546, L123-L126.

Chyba, C.F. \& Sagan, C. (1992). Nature 355, 125-132.

Chyba, C.F., Thomas, P.J., Brookshaw, L. \& Sagan, C. (1990). Science 249, 366-373.

Cody, G.D. \& Alexander, C.M. O'D (2005). Geochim. Cosmochim. Acta 69, 1085-1097.

Cronin, J.R. \& Chang, S. (1993). The Chemistry of Life's Origins. eds Greenberg, J.M., Mendoze-Gómez, C.X. \& Pirronello, V., pp. 209-258. Kluwer, Dordrecht.

Cronin, J.R., Pizzarello, S. \& Frye, J.S. (1987). Geochim. Cosmochim. Acta 51, 299-303.

Cruikshank, D.P. (2008). Organic Matter in Space (IAU Symp. 251). eds Kwok, S. \& Sandford, V., pp. 285-292. Cambridge University Press.

Cruikshank, D.P. et al. (2008). Icarus 193, 334-343.

Deamer, D.W. (1997). Microbiol. Mol. Bio. Reviews 61, 239-261.

Duley, W.W. \& Williams, D.A. (1981). Mon. Not. R. Astron. Soc. 196, 269-274.

Ehrenfreund, P. \& Charnley, S.B. (2000). Ann. Rev. Astron. Astrophys. 38, 427-483.

Ehrenfreund, P., Rasmussen, S., Cleaves, J. \& Chen, L. (2006). Astrobiology 6, 490-520.

Ehrenfreund, P., Robert, F., d'Henecourt, L. \& Behar, F. (1991). Astron. Astrophys. 252, 712-717.

Ehrenfreund, P. et al. (2002). Rep. Prog. Phys. 65, 1427-1487.

Flynn, G.J., Keller, L.P., Feser, M., Wirick, S. \& Jacobsen, C. (2003). Geochimica Cosmochimica Acta 67, 4791-4806.

Garcia-Lario, P., Manchado, A., Ulla, A. \& Manteiga, M. (1999). Astrophys. J. 513, 941-946.

Gradie, J. \& Veverka, J. (1980). Nature 283, 840-842.

Guillois, O., Nenner, I., Papoular, R. \& Reynaud, C. (1996). Astrophys. J. 464, 810-817.

Haldane, J.B.S. (1929). The Origin of Life, Rationalist Annu. 148, 3-10.

Harris, S.J. \& Weiner, A.M. (1985). Ann. Rev. Phys. Chem. 36, 31-52.

Henning, T. (2009). Interstellar Dust from Astronomical Observations to Fundamental Studies, Boulanger, F., Joblin, C., Jones, A. \& Madden, S., EAS Publications Series 35, pp. 103-114. EAS.

Herlin, N., Boh, I., Reynaud, C., Cauchetier, M., Galvez, A. \& Rouzaud, J.N. (1998). Astron. Astrophys. 330, 1127-1135. 
Hoyle, F. \& Wickramsinghe, N.C. (1977). Nature 268, 610-612.

Hrivnak, B.J., Geballe, T.R. \& Kwok, S. (2007). Astrophys. J. 662, 1059-1066.

Hrivnak, B.J., Kwok, S. \& Su, K.Y.L. (1999). Astrophys. J. 524, 849-856.

Hrivnak, B.J., Volk, K. \& Kwok, S. (2000). Astrophys. J. 535, 275-292.

Hudgins, D.M., Bauschlicher, C.W. \& Allamandola, L.J. (2005). Astrophys. J. 632, 316-332.

Jäger, C., Mutschke, H., Henning, Th. \& Huisken, F. (2008). Astrophys. J. 689, 249-259.

Jones, A.P., Duley, W.W. \& Williams, D.A. (1990). Q.J.R. Astron. Soc. 31, $567-582$.

Jones, A.P., Tielens, A.G.G.M. \& Hollenbach, D.J. (1996). Astrophys. J. 469, 740-764.

Kerridge, J.F. (1999). Space Sci. Rev. 90, 275-288.

Kring, D.A. (2008). NLSI Lunar Science Conference, held July 20-23, 2008 at NASA Ames Research Centre, Moffett Field, California, LPI Contribution No. 1415, abstract No. 2140.

Kwok, S. (1987). Phys. Reports 156(3), 113-146.

Kwok, S. (1990). Mon. Not. R. Astron. Soc. 244, 179-183.

Kwok, S. (2000). The Origin and Evolution of Planetary Nebulae. Cambridge University Press.

Kwok, S., Volk, K. \& Bernath, P. (2001). Astrophys. J. 554, L87-L90.

Kwok, S., Volk, K. \& Bidelman, W.P. (1997). Astrophys. J. Suppl. 112, $557-584$.

Kwok, S., Volk, K. \& Hrivnak, B.J. (1999). Astron. Astrophys. 350, L35-L38.

Lewis, R.S., Tang, M., Wacker, J.F., Anders, E. \& Steel, E. (1987). Nature 326, 160-162.

Martins, Z., Botta, O., Fogel, M. L., Sephton, M. A., Glavin, D. P., Watson, J. S., Dworkin, J. P., Schwartz, A. W., Ehrenfreund, P. (2008). Earth Planet. Sci. Lett. 270, 130-136.
Mennella, V., Colangeli, L., Palumbo, P., Rotundi, A., Schutte, W. \& Bussoletti, E. (1996). Astrophys. J. 464, L191-L94.

Messenger, S., Keller, L.P., Stadermann, F.J., Walker, R.M. \& Zinner, E. (2003). Science 300, 105-108.

Miller, S.L. (1953). Science 117, 528-529.

Miller, S.L. \& Urey, H.C. (1959). Science 130, 245.

Nagashima, K., Krot, A.N. \& Yurimoto, H. (2004). Nature 428, 921-924.

Nakamura-Messenger, K., Messenger, S., Keller, L.P., Clemett, S.J. \& Zolensky, M.E. (2006). Science 314, 1439-1442.

Nguyen, A.N. \& Zinner, E. (2004). Science 303, 1496-1499.

Nittler, L.R., Alexander, C.M.O'D., Gzo, X., Walker, R.M. \& Zinner, E. (1997). Astrophys. J. 483, 475-495.

Oparin, A.I. (1938). The Origin of Life. MacMillan, New York.

Papoular, R. (2001). Astron. Astrophys. 378, 597-607.

Pendleton, Y.J. \& Allamandola, L.J. (2002). Astrophys. J. Suppl. 138, 75-98.

Posch, T., Kerschbaum, F., Mutschke, H., Fabian, D., Dorschner, J. \& Hron, J. (1999). Astron. Astrophys. 352, 609-618.

Posch, Th., Kerschbaum, F., Mutschke, H., Dorschner, J. \& Jäger, C. (2002). Astron. Astrophys. 393, L7-L10.

Russell, R.W., Soifer, B.T. \& Willner, S.P. (1977). Astrophys. J. 217, L149-L153.

Sakata, A., Wada, S., Onaka, T. \& Tokunaga, A.T. (1987). Astrophys. J., 320, L63-L67.

Sakata, A., Wada, S., Tanabé, T. \& Onaka, T. (1984). Astrophys. J. 287, L51-L54.

Schönberner, D. (1983). Astrophys. J. 272, 708-714

Suess, H.E. (1965). Ann. Rev. Astron. Astrophys. 3, 217-234.

Volk, K.M. \& Kwok, S. (1989). Astrophys. J., 342, 345-363.

Volk, K., Xiong, G.-Z. \& Kwok, S. (2000). Astrophys. J. 530, 408-417.

Wooden, D.H., Harker, D.E., Woodward, C.E., Butner, H.M., Koike, C., Witteborn, F.C. \& McMurty, C.W. (1999). Astrophys. J. 517, 1034-1058. 